\title{
Enhanced Rare Pion Decays from a Model of MeV Dark Matter
}

\author{
Yonatan Kahn, ${ }^{1}$ Michael Schmitt,${ }^{1}$ and Tim M.P. Tait ${ }^{1,2}$ \\ ${ }^{1}$ Northwestern University, Department of Physics and Astronomy, 2145 Sheridan Road, Evanston, IL 60208, USA \\ ${ }^{2}$ HEP Division, Argonne National Lab, Argonne IL 60439 USA
}

(Dated: August 12, 2008)

\begin{abstract}
A model has been proposed in which neutral scalar particles $\chi$, of mass $1-10 \mathrm{MeV}$, annihilate through the exchange of a light vector boson $U$, of mass $10-100 \mathrm{MeV}$, to produce the $511 \mathrm{keV}$ line observed emanating from the center of the galaxy. The $\chi$ interacts weakly with normal matter and is a viable dark matter candidate. If the $U$-boson couples to quarks as well as to electrons, it could enhance the branching ratio for the rare decay $\pi^{0} \rightarrow e^{+} e^{-}$. A recent measurement by the $\mathrm{KTeV}$ Collaboration lies three standard deviations above a prediction by Dorokhov and Ivanov, and we relate this excess to the couplings of the $U$-boson. The values are consistent with other constraints and considerations. We make some comments on possible improvements in the data.
\end{abstract}

PACS numbers: $12.60 . \mathrm{Cn}, 13.20 . \mathrm{Cz}, 14.70 . \mathrm{Pw}$

\section{INTRODUCTION}

Astronomical observations over the past century have shown that approximately $20 \%$ of the universe is made of dark matter. Since dark matter has only been detected through its gravitational interactions, several of its properties, including the mass of dark matter particles and their interactions with Standard Model (SM) particles, remain completely unknown. While the WIMP (Weakly Interacting Massive Particle) with mass of order the electroweak scale is arguably the most popular candidate at this time, the possibility that dark matter could be lighter than $100 \mathrm{MeV}$ has attracted much attention recently. A model proposed by Boehm et al. 1] postulates a neutral scalar dark matter particle $\chi$ with mass $1-10 \mathrm{MeV}$ [2], which annihilates to produce electron/positron pairs: $\chi \chi \rightarrow e^{+} e^{-}$(alternately, $\chi$ could be a fermion [3]). The excess positrons produced in this annihilation reaction could be responsible for the bright $511 \mathrm{keV}$ line emanating from the center of the galaxy [4], as more conventional astrophysical explanations have failed to explain both the intensity and shape of this line. Boehm proposes two particles which mediate $\chi$ annihilation: a neutral vector boson $U$, with mass $m_{U} \sim 10-100 \mathrm{MeV}$, and a heavy fermion $F^{ \pm}$with mass $>100 \mathrm{GeV}$. The $U$ boson is needed to explain the relic dark matter density, while the $F$ fermion is necessary to account for the observed rate of positron annihilation in the galactic center [5]. For early incarnations of the $U$ boson, see [6].

The rare decay $\pi^{0} \rightarrow e^{+} e^{-}$has long posed an interesting problem in the theory of strong interactions. Since it is suppressed at tree-level, the rate is very small, which provides an opening for indirect effects of new physics to appear. A new precise measurement of $B\left(\pi^{0} \rightarrow e^{+} e^{-}\right)$by the $\mathrm{KTeV}$ Collaboration exceeds the most recent theoretical calculation. In this Letter, we examine the possibility that this excess can be explained by the exchange of an off-shell $U$ boson of the type proposed in the light dark matter model, and we compare the constraints obtained on the coupling constants to other constraints.

\section{PION DECAY}

The tree-level decay $\pi^{0} \rightarrow \gamma^{*} \rightarrow e^{+} e^{-}$is forbidden because the pion is a pseudo-scalar particle and the massless photon has no longitudinal component. Consequently, the lowest-order SM contribution is a one-loop process with a two-photon intermediate state. The suppression of the amplitude by a factor of $\alpha^{2}$ and by helicity conservation leads to an extremely small decay width, thus allowing for even tiny effects of new physics to be detectable.

The KTeV-E779 Collaboration recently published a new measurement of $B\left(\pi^{0} \rightarrow e^{+} e^{-}\right)$[7]. They normalized this branching ratio to the Dalitz decay mode $B\left(\pi^{0} \rightarrow e^{+} e^{-} \gamma\right)$, taking both sets of decays from a large sample of $K_{L} \rightarrow 3 \pi^{0}$ events. The similarity of the signal and normalization channels serves to minimize acceptance and efficiency uncertainties, and the remaining systematic uncertainties are mainly external, deriving from the rate of Dalitz decays and the parametrization of the pion form factor. The $\mathrm{KTeV}$ Collaboration report the value

$$
B^{\text {meas }}\left(\pi^{0} \rightarrow e^{+} e^{-}\right)=(7.48 \pm 0.29 \pm 0.25) \times 10^{-8}
$$

after extrapolating from the selected to the entire kinematic region. The first error is from data statistics alone, while the second is the total systematic error.

The most recent theoretical estimate of the $\pi^{0} \rightarrow e^{+} e^{-}$ width was completed by Dorokhov and Ivanov, who obtained

$$
B^{\mathrm{SM}}\left(\pi^{0} \rightarrow e^{+} e^{-}\right)=(6.2 \pm 0.1) \times 10^{-8},
$$

and noted the discrepancy with respect to the $\mathrm{KTeV}$ measurement [8]. The dominant theoretical uncertainty comes from the hadronic form factors, expressed through a dispersion relation in terms of a subtraction constant. The authors of [8] estimate the value of the subtraction constant by using an assumed monopole functional form and comparing with CLEO data. They further compare their estimate with results derived from the operator product expansion, QCD sum rules, generalized 
vector meson dominance, and a non-local constituent quark model. All results agree with each other within the quoted uncertainties.

The excess of $B^{\text {meas }}$ over $B^{\mathrm{SM}}$ suggests that non-SM processes may be contributing to this rare decay. If the $U$ boson couples to quarks as well as electrons, the lowestorder contribution to $\pi^{0} \rightarrow e^{+} e^{-}$would come from the tree-level process $\pi^{0} \rightarrow U^{\star} \rightarrow e^{+} e^{-}$. The smallness of this contribution would be explained by very small values of the coupling constants, which are, in fact, natural in the light dark matter model [1, 9].

The $U$ boson coupling to quarks and electrons can be written in terms of vector and axial-vector components,

$$
\begin{aligned}
\mathcal{L} \supset & U_{\mu}\left\{\bar{u} \gamma^{\mu}\left(g_{V}^{u}+\gamma_{5} g_{A}^{u}\right) u+\bar{d} \gamma^{\mu}\left(g_{V}^{d}+\gamma_{5} g_{A}^{d}\right) d\right. \\
& \left.+\bar{e} \gamma^{\mu}\left(g_{V}^{e}+\gamma_{5} g_{A}^{e}\right) e\right\}
\end{aligned}
$$

where $u$ and $d$ are the up and down quark fields, and $e$ is the electron field. It is not necessary to have familyuniversal couplings, and in fact we will assume that couplings to the second and third generations are suppressed. To respect the unitary bound in the ultra-violet, the $U$ should correspond to a local $U(1)_{U}$ symmetry, which is spontaneously broken. One might worry that the presence of axial vector couplings implies that the Yukawa interactions between $u, d$, and $e$ and the Higgs responsible for generating fermion masses are not symmetric under $U(1)_{U}$. However, given the tiny $u, d$, and $e$ masses compared to the electroweak scale, it is easy to accommodate them from effective higher dimensional operators induced by high mass states.

At tree level, the contribution to $\pi^{0} \rightarrow e^{+} e^{-}$is mediated by an off-shell $U$ boson, as depicted in Fig. 1. The $U$ boson contribution to the matrix element is given by

$$
\mathcal{M}_{U}=\frac{\left(g_{A}^{d}-g_{A}^{u}\right) g_{A}^{e} f_{\pi}}{m_{U}^{2}}\left[\bar{u} \gamma^{\mu} \gamma_{5} v\right] p_{\mu}
$$

where $m_{e}$, and $m_{U}$ are the electron, and $U$-boson masses, $f_{\pi}$ is the pion decay constant, and $p_{\mu}$ is the $\pi^{0}$ fourmomentum, $p^{2}=m_{\pi}^{2}$. (See the Appendix ${ }_{\square}$ for details).

To obtain the full amplitude for $\pi^{0} \rightarrow e^{+} e^{-}$, the $U$ boson matrix element is combined with the Standard Model amplitude for $\pi^{0} \rightarrow e^{+} e^{-}$[8] and summed over the outgoing electron and positron spins. The partial width $\pi^{0} \rightarrow e^{+} e^{-}$is computed from the expression for the two-body decay,

$$
\Gamma=\frac{|\vec{p}|}{8 \pi m_{\pi}^{2}} \overline{\left|\mathcal{M}_{S M}+\mathcal{M}_{U}\right|^{2}}
$$

where $|\vec{p}|$ is the three-momentum of one of the outgoing particles, and is equal to approximately $m_{\pi} / 2$, neglecting the electron mass.

\section{BOUNDS ON U-QUARK COUPLINGS}

We interpret the positive difference $B^{\text {meas }}-B^{\mathrm{SM}}=$ $(1.3 \pm 0.4) \times 10^{-8}$ as the contribution of $\mathcal{M}_{U}$ in Eq. (3).

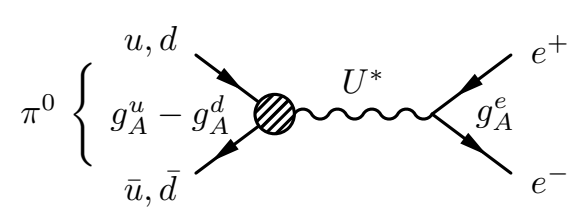

FIG. 1: Feynman diagram for $\pi^{0} \rightarrow e^{+} e^{-}$.

Taking the known pion and electron masses, $f_{\pi^{0}}=130 \pm$ $5 \mathrm{MeV}$ and $\tau_{\pi^{0}}=(84 \pm 6) \times 10^{-18} \mathrm{~s}[10]$, we find

$$
\frac{\left(g_{A}^{u}-g_{A}^{d}\right) g_{A}^{e}}{m_{U}^{2}}=(4.0 \pm 1.8) \times 10^{-10} \mathrm{MeV}^{-2}
$$

In order to make contact with other constraints on this model, we assume, as an illustration, that the electron coupling and the difference in quark couplings are equal, i.e., $g_{A}^{u}-g_{A}^{d}=g_{A}^{e} \equiv g_{A}$. This choice is arbitrary, but one might naturally expect such a relation to hold within an order of magnitude; a more precise relation requires a specific model for the fermion charges under $U(1)_{U}$, which is beyond the scope of this Letter. With this assumption,

$$
g_{A}=2.0_{-0.5}^{+0.4} \times 10^{-4} \times\left(\frac{m_{U}}{10 \mathrm{MeV}}\right)
$$

where the asymmetric error bars come from taking the square root of Eq. (4). Fig. 2] shows this constraint as a thick line labeled " $\pi^{0}$ ". If a given model specifies a different relation between $g_{A}^{u}-g_{A}^{d}$ and $g_{A}^{e}$, then this line will move vertically in the plot.

Fayet has derived other bounds on the coupling of $U$ bosons to quarks and leptons from a variety of processes [9], and some of these are shown in Fig. 2. The dashed line labeled " $(g-2) e$ " indicates his constraints on the axial coupling of $U$ to electrons derived from measurements of the anomalous magnetic moment of the electron; the region above this line is excluded. Constraints from kaon decays, as well as $(g-2)_{\mu}[9]$, can be evaded if we assume that couplings to second and third generation fermions are suppressed. Neutrinoelectron scattering can provide a relatively severe constraint [9], but may be evaded if the coupling to electrons is largely right-handed. Finally, the three solid lines labeled "1 MeV," etc., show constraints on the total $U-e$ coupling $f_{\text {tot }}=\sqrt{\left(f_{V}^{e}\right)^{2}+\left(f_{A}^{e}\right)^{2}}$ from the dark matter relic density [9], assuming $C_{\chi}=1$, for three hypothetical values of the $\chi$ mass. The regions above these lines correspond to smaller values of $C_{\chi}$.

The curves in Fig. 2 show that our values for the couplings of the $U$-boson to light quarks and leptons are interesting in the context of the light dark matter model, falling in the same order-of-magnitude as other constraints. Since $\mathcal{M}_{\mathcal{U}}$ depends on a set of coupling constants different from the other constraints, the rare decay $\pi^{0} \rightarrow e^{+} e^{-}$provides a different view of the phenomenology of the light $U$ boson. 


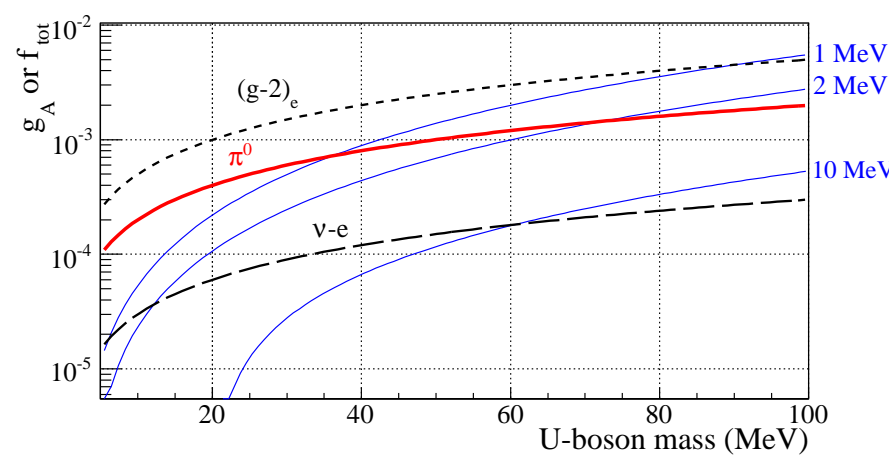

FIG. 2: Constraints on the couplings as a function of $m_{U}$. The thick (red) line labeled " $\pi^{0}$ " shows our result Eq. (4). The dotted line comes from constraints on $(g-2)_{e}$ and the dashed line from $\nu$ - $e$ scattering [9]. The solid (blue) lines labeled with " $n \mathrm{MeV}$ " are based on relic density calculations [9].

We note that the partial width for the electroweak interaction process $\pi^{0} \rightarrow Z^{\star} \rightarrow e^{+} e^{-}$is identical to Eq. (2) with $m_{U}$ replaced by $m_{Z}$ and $g_{A}^{u, d, e}$ replaced by the analogous weak couplings; even with $g_{A}^{u, d, e}$ as small $10^{-4}$, the large mass of the $Z$ boson renders this electroweak process completely negligible compared to the $U$-mediated decay.

\section{POSSIBLE IMPROVEMENTS}

The excess depends on one precise measurement and one precise calculation, and has a significance of only $3 \sigma$. Improvements to this situation would help sharpen the discussion.

The $\mathrm{KTeV}$ measurement depends crucially on the branching ratio for Dalitz decays, which was last measured in 1981 [1] to a precision of $3 \%$. Combined with a bubble chamber measurement from 1961 [12], the world's best value for this basic benchmark decay is precise at the level of $2.7 \%$ [10]. We speculate that modern experiments could provide a more precise measurement. For example, Na48/2 has reconstructed $9.1 \times 10^{7}$ decays $K^{ \pm} \rightarrow \pi^{ \pm} 2 \pi^{0}$ as a key part of their program to study $\mathrm{CP}$ violations in charged kaon systems [13]. Their trigger does not appear to have suppressed Dalitz decays. As these decays take place in a long vacuum tube, there is no background from converted photons. The resolution on photon energies and position is excellent, allowing a direct reconstruction of the vertex position. Combined with the charged pion, the mass resolution is $0.9 \mathrm{MeV} / c^{2}$. Track and photon reconstruction efficiencies have been measured directly from the data. Perhaps the acceptance ratio for one charged track plus five electromagnetic clusters over one charged track plus four electromagnetic clusters could be estimated by simulations at the $1 \%$ level. If so, a much better measurement of $B\left(\pi^{0} \rightarrow e^{+} e^{-} \gamma\right)$ would appear to be possible. Clearly, other experiments might endeavor to update this branching ratio measurement.

Another approach to testing the SM with better precision might be to compare the measurement of the ratio of branching ratios, $B\left(\pi^{0} \rightarrow e^{+} e^{-}\right) / B\left(\pi^{0} \rightarrow e^{+} e^{-} \gamma\right)$, to the theoretical prediction. Clearly this ratio is experimentally much more precise, and we expect the theoretical uncertainties coming from the hadronic form factor to be reduced as well.

Of course, a direct measurement of $B\left(\pi^{0} \rightarrow e^{+} e^{-}\right)$by another experiment would be extremely interesting.

We considered possible enhancements to leptonic $\eta$ decays provided by a light $U$-boson. Following the same calculation as above, we find

$$
\mathcal{M}_{U}=\frac{3\left(g_{A}^{\ell}\left(g_{A}^{u}+g_{A}^{d}-2 g_{A}^{s}\right) f_{\eta}^{8}\right)}{m_{U}^{2}}\left[\bar{u} \gamma^{\mu} \gamma_{5} v\right] p_{\mu}
$$

Assuming that the couplings are of the same order as indicated in Eq. (5), we find $B\left(\eta \rightarrow e^{+} e^{-}\right) \sim 10^{-9}$, which is of the same order as the improved unitary bound [8], and much smaller than the experimental bound. $\eta$ mesons are heavy enough to decay to muons, and we estimate $B\left(\eta \rightarrow \mu^{+} \mu^{-}\right) \sim 2.0 \times 10^{-5}$. This prediction is nearly an order of magnitude larger than the measured value [14], $B\left(\eta \rightarrow \mu^{+} \mu^{-}\right)=(5.7 \pm 0.9) \times 10^{-6}$. If the $U$-boson exists, then its couplings to muons must be smaller than its couplings to electrons, or the combination of quark axial couplings $\left(g_{A}^{u}+g_{A}^{d}-2 g_{A}^{s}\right)$ is smaller than $\left(g_{A}^{u}-g_{A}^{d}\right)$.

\section{SUMMARY}

The $3 \sigma$ excess of the $\mathrm{KTeV}$ measurement of $B\left(\pi^{0} \rightarrow\right.$ $\left.e^{+} e^{-}\right)$over the most recent calculation by Dorokhov and Ivanov prompts considerations of a new physics contribution to this SM-suppressed decay. The light neutral vector $U$ boson proposed in the light dark matter model provides a good basis for calculating such a contribution. We carried out such a calculation and find that couplings of the $U$-boson to electrons and light quarks should be on the order of $2 \times 10^{-4}$, for $m_{U}=10 \mathrm{MeV}$. Such small couplings are consistent with the expectations of the light dark matter model, and with other constraints coming from leptonic processes.

\section{Appendix: Matrix element calculation}

The $U$-boson exchange couples to both vector and axial vector currents of quarks, evaluated between a $\pi^{0}$ state and the vacuum (see Figure 1),

$$
\left\langle 0\left|\left\{\bar{u} \gamma^{\mu}\left(g_{V}^{u}+\gamma_{5} g_{A}^{u}\right) u+\bar{d} \gamma^{\mu}\left(g_{V}^{d}+\gamma_{5} g_{A}^{d}\right) d\right\}\right| \pi^{0}\right\rangle .
$$

We can reorganize the four terms into combinations with definite parity and transformation under strong iso-spin, $S U(2)_{V}$. From there, it is easy to see that the only combination which contributes is the current proportional to 
the third component of axial iso-spin,

$$
\left\langle 0\left|\frac{1}{2}\left\{\bar{u} \gamma^{\mu} \gamma_{5} u-\bar{d} \gamma^{\mu} \gamma_{5} d\right\}\right| \pi^{0}\right\rangle \equiv f_{\pi} p^{\mu} .
$$

where $p^{\mu}=p_{e^{+}}^{\mu}+p_{e^{-}}^{\mu}$ is the momentum of the pion. Note that the pion state singles out the axial couplings in the combination $g_{A}^{u}-g_{A}^{d}$.

The hadronic vertex is contracted through the $U$ boson, which also carries momentum $p$ into the leptonic vertex,

$$
\mathcal{M}=\frac{\left(g_{A}^{u}-g_{A}^{d}\right) g_{A}^{e}}{m_{\pi}^{2}-m_{U}^{2}}\left[\bar{u} \gamma^{\mu} \gamma_{5} v\right]\left[g_{\mu \nu}-\frac{p_{\mu} p_{\nu}}{m_{U}^{2}}\right] f_{\pi} p^{\nu}
$$

where $u$ and $v$ are the electron and positron spinors, respectively. For an on-shell pion, $p^{\mu} p_{\mu}=m_{\pi}^{2}$, and we obtain

$$
\mathcal{M}=\frac{\left(g_{A}^{d}-g_{A}^{u}\right) g_{A}^{e} f_{\pi}}{m_{U}^{2}}\left[\bar{u} \gamma^{\mu} \gamma_{5} v\right] p_{\mu}
$$

\section{Acknowledgments}

We thank Céline Boehm and for stimulating discussions and Mayda Velasco for interesting discussions of measurements of light meson decays.

Research at Argonne National Laboratory is supported in part by the Department of Energy under contract DEAC02-06CH11357, and the work by Y.K. and M.S. is supported by contract DE-FG02-91ER40684.
[1] C. Boehm and P. Fayet, Nucl. Phys. B683 219 (2004); C. Boehm, T.A. Ensslin and J. Silk, J. Phys. G30 279 (2004);

C. Boehm, D. Hooper, J. Silk and M. Casse, Phys. Rev. Lett. 92101301 (2004);

C. Boehm and Y. Ascasibar, Phys. Rev. D70 115013 (2004)

[2] J.F. Beacom and H. Yuksel, Phys. Rev. Lett. 97071102 (2006);

J.F. Beacom, N.F. Bell, and G. Bertone, Phys. Rev. Lett. 94171301 (2005)

[3] Y. Rasera, R. Teyssier, P. Sizun, B. Cordier, J. Paul, M. Casse and P. Fayet, Phys. Rev. D 73, 103518 (2006) arXiv:astro-ph/0507707.

[4] G. Weidenspointner et al. , Nature 451159 (2008);

J. Knodsleder et al. , Astron. Astrophys. 441513 (2005); ibid., 411 L457 (2003);

W.N. Johnson III, F. R. Harnden, Jr. and R.C. Haymes, Ap. J. 172 L1 (1972);
M. Leventhal, C.J. MacCallum and P. D. Stang, Ap. J. 225 L11 (1978);

L.X. Cheng, et al. , Ap. J. (Letters) 481 L43 (1997);

W.R. Purcell, et al. , Ap. J. 491725 (1997)

[5] Y. Ascasibar, P. Jean, C. Boehm and J. Knoedlseder, Mon. Not. Roy. Astron. Soc. 3681695 (2006)

[6] P. Fayet, Nucl. Phys. B 187, 184 (1981).

[7] KTeV Collab. [E. Abouzaid et al. ], Phys. Rev. D75 012004 (2007)

[8] A. Dorokhov and M. Ivanov, Phys. Rev. D75 114007 (2007)

[9] Pierre Fayet, Phys. Rev. D75 115017 (2007); ibid., D74 054034 (2006)

[10] W.-M. Yao et al. [PDG Collab.], J. Phys. G33 1 (2006)

[11] M. A. Schardt et al. , Phys. Rev. D 23, 639 (1981).

[12] N. P. Samios et al. , Phys. Rev. 121, 275 (1961).

[13] Na48/2 Collab., [J. R. Batley et al. ], arXiv:0707.0697

[14] R. Abegg et al., Phys. Rev. D50, 92 (1994). 